\title{
Isolated limb infusion chemotherapy for melanoma: an overview of early experience at the Adelaide Melanoma Unit
}

This article was published in the following Dove Press journal:

Cancer Management and Research

19 August 2013

Number of times this article has been viewed

\section{Mitchell H Giles' \\ Brendon J Coventry ${ }^{2}$}

'Adelaide Melanoma Unit, ${ }^{2}$ Discipline of Surgery, The University of Adelaide, Royal Adelaide Hospital Adelaide, SA, Australia
Correspondence: BJ Coventry Adelaide Melanoma Unit, Discipline of Surgery, The University of Adelaide, Level 5 EHB, Royal Adelaide Hospital Adelaide, SA 5000, Australia Email brendon.coventry@adelaide.edu.au
Background: Isolated limb infusion (ILI) using cytotoxic agents has been demonstrated to be an effective and less invasive alternative modality than isolated limb perfusion for the treatment of melanoma localized to a limb. Percutaneous catheters were inserted into the axial artery and vein of the affected limb while using a pneumatic cuff to restrict limb vascular flow proximally to "isolate" the limb from the body and enable delivery of high-dose intra-arterial chemotherapy selectively to the limb. The ILI technique was developed at the Sydney Melanoma Unit (now renamed the Melanoma Institute Australia), and only a few other centers have reported separate results. We report our early results using the ILI technique for management of locally recurrent surgically nonresectable melanoma.

Methods and results: Twenty-eight ILI procedures were performed in 20 patients treated with one or more procedures between 1997 and 2007. Patient parameters and clinical responses were evaluated. The median follow-up duration was 15.9 months after the first ILI, with an overall response rate after one or more infusions of $70 \%$, of which $35 \%$ were complete responders and $35 \%$ were partial responders, with a further $20 \%$ showing stable disease, giving a "clinically significant" response rate of $90 \%$. After one ILI $(n=20)$, the overall response rate was $70 \%$, with $20 \%$ complete responders and $50 \%$ partial responders, and $20 \%$ with stable disease. Low limb toxicities were generally observed, and no amputations were required.

Conclusion: ILI chemotherapy is a useful technique, which can be readily repeated for control of melanoma in the limb. It is generally well tolerated, and is capable of achieving a cure, delayed progression, or effective palliation in selected cases. The longest survivors in this series were 8 and 10 years from the last ILI.

Keywords: metastatic melanoma, melphalan, actinomycin-D, regional therapy, intra-arterial infusion

\section{Introduction}

The incidence of melanoma in Australian men and women increased by $42 \%$ and $18 \%$, respectively, from 1991 to $2009,{ }^{1}$ bringing with it a corresponding increase in the number of patients presenting with local recurrences following initial surgical excision. A study from the Sydney Melanoma Unit examining 4704 American Joint Committee on Cancer stage I and II patients reported a recurrence rate of $18.6 \%$ in 873 patients, of which $10.9 \%$ were local and 9.9\% in-transit in type, over a median follow-up duration of 5.3 years after initial excision of primary melanoma. ${ }^{2}$ Many of these are related to risk factors of the primary melanoma, such as high Breslow thickness or ulceration.

The modalities of treatment for patients with localized recurrence of limb melanoma are numerous, including local surgical excision, laser ablation, local injection, 
cryotherapy, and vaccine therapy. However, when metastases are numerous or bulky, these options are limited and other techniques should be considered, such as regional chemotherapy, radiotherapy, or systemic chemotherapy. Chemotherapy doses achievable using standard systemic chemotherapy are lowered due to risk of systemic side effects; however, higher doses of chemotherapy can be delivered to the limb if it is vascularly "isolated" from the systemic circulation. Isolated limb perfusion was developed in the $1950 \mathrm{~s}^{3}$ and used in many centers worldwide, but carries higher risks of morbidity and mortality because of its highly invasive nature. It requires use of vascular dissection and clamping as well as an extracorporeal bypass pump circuit, making it a complex and costly procedure. Overall response rates reported using isolated limb perfusion range from $71 \%$ to $88 \%$, with complete response rates of $12 \%-73 \% .^{4-11}$ This wide range in complete response rates is likely related to differences in patient selection factors between studies. Known complications after isolated limb perfusion include compartment syndrome, neuropathy, infection, and arterial or venous thrombosis. ${ }^{4-18}$

Over the last two decades, isolated limb infusion (ILI) has been used at the Sydney Melanoma Unit (now known as the Melanoma Institute Australia) as an alternative to isolated limb perfusion. ILI has the advantage of being a much simpler procedure, circumventing the need for complex bypass, extensive surgical dissection, and long operating times, whilst being well tolerated and, if required, readily repeated, even in the elderly. High doses of chemotherapy, above systemically tolerable levels, can be delivered selectively to the affected limb, without leakage into the systemic circulation. Clinical response rates using ILI have been demonstrated to be comparable with those obtained using isolated limb perfusion. ${ }^{15,16}$

\section{Patients and methods}

Informed consent was obtained from all patients and the studies were approved by the Royal Adelaide Hospital human research ethics committee. Analysis was conducted on data from a total of 28 ILI procedures that were performed between July 1997 and May 2007 in 20 patients at the Royal Adelaide Hospital. The indication for treatment was recurrent lower or upper limb melanoma for which surgery was not feasible, presenting with either satellite or in-transit metastases and with or without previous surgically resected lymph node involvement. Patients with stage IV disease were treated for palliative purposes and were also included in this study to assess limb disease responses.
The technique used for ILI is shown in Figure 1 and have been detailed elsewhere. ${ }^{15}$ In summary, a nonoxygenated low-flow extracorporeal circuit was created using percutaneously inserted arterial and venous catheters (6 French and 8 French, respectively). Under general anesthesia, the patient was systemically heparinized, followed by a rapid intraarterial bolus injection of papaverine, and then isolation of the disease-affected limb using an inflatable tourniquet, placed proximally, high on the limb. Rapid intra-arterial infusion of melphalan and actinomycin D followed. Using a high-flow, three-way stopcock valve, the infusate was manually circulated using a $60 \mathrm{~mL}$ syringe for up to 30 minutes. Blood warming coils were incorporated into this circuit to maintain limb temperature, and hot-air blankets were used to cover the patient's limbs and body. Intramuscular and subcutaneous temperature probes were inserted into the limb for monitoring the temperatures throughout the procedure. Following circulation, the infusate was drained from the isolated circuit, discarded, and replaced by Hartmann's solution to wash out the intravascular chemotherapeutic agent. Protamine was administered to reverse the heparinization and the catheters were removed. Pressure was applied to the puncture sites, followed by reversal of the general anesthetic.

Patients were restricted to bed rest with elevation of the treated limb for 48 hours following the procedure. Hourly limb observation, daily creatine kinase measurements, and limb toxicity assessments were made using the scale published by Wieberdink et $\mathrm{al}^{19}$ (see Table 1).

Patients' responses were clinically defined by World Health Organization criteria for reporting cancer treatment, where determinant measurements were taken not less than 4 weeks apart. ${ }^{20} \mathrm{~A}$ complete response was defined by a $100 \%$ reduction in tumor mass; a partial response was a reduction in tumor mass by over $50 \%$; stable disease was a reduction of less than $50 \%$ or an increase in mass of no more than 25\%; whilst progressive disease was defined as any increase in mass greater than $25 \%$. Overall response was defined as the combined complete response and partial response rates. Response duration was defined as the time elapsed between the ILI procedure and the development of new or progressive disease. Assessment of response was conducted by the same clinician on each occasion with measurement comparisons pre-ILI and on subsequent follow-up.

The results were analyzed as follows: assessment after the initial ILI procedure for all patients to assess initial 

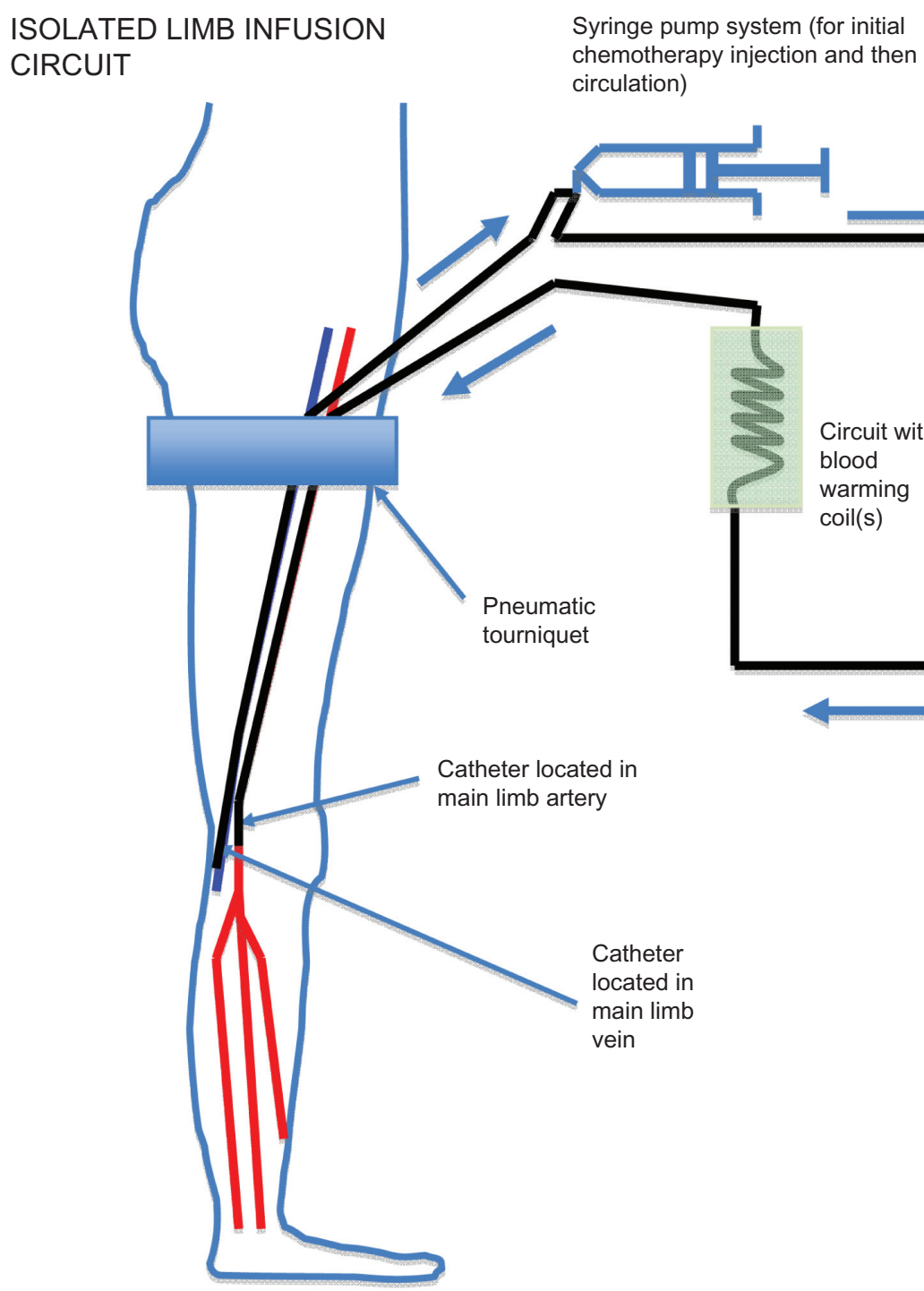

Figure I Isolated limb infusion circuit in a lower limb showing positioning of the tourniquet, arterial and venous catheters inserted in the ipsilateral (or contralateral) groin to reach approximately the level of the popliteal artery and vein, respectively, and the extracorporeal warmed circuit using a hand-driven syringe pump.

outcome ( $\mathrm{n}=20)$; collectively for all patients, regardless of the number of ILI procedures performed, to assess overall outcome $(n=20)$; patients receiving a single ILI procedure alone $(\mathrm{n}=15)$; assessment of all ILI treatment episodes for all patients; and assessment of the toxicity of each ILI procedure $(n=28)$.

\section{Statistical analysis}

An ordinal regression model was used to determine any association between patient characteristics and clinical response. A statistically significant difference was assumed at a probability value of $<0.05$. Statistical analyses were performed using Statistical Package for Social Sciences version 13 for MAC OSX (SPSS Inc, Chicago, IL, USA).

\section{Results}

\section{Patient data}

Between July 1997 and May 2007, 28 ILI procedures were performed in 20 patients at the Royal Adelaide Hospital. Fifteen patients underwent a single ILI procedure, two

Table I Wieberdink grades ${ }^{19}$

\begin{tabular}{ll}
\hline Grade I & No visible effect \\
Grade II & Slight erythema and/or edema \\
Grade III & $\begin{array}{l}\text { Considerable erythema and/or edema } \\
\text { Grade IV }\end{array}$ \\
& $\begin{array}{l}\text { Extensive epidermolysis and/or obvious damage to deep } \\
\text { tissues with a threatened or actual compartment syndrome }\end{array}$ \\
Grade V & Severe tissue damage necessitating amputation \\
\hline
\end{tabular}

Notes: Reprinted from Eur J Cancer Clin Oncol, 18, Wieberdink J, Benckhuysen C, Braat RP, et al, Dosimetry in isolation perfusion of the limbs by assessment of perfused tissue volume and grading of toxic tissue reactions, 905-910. Copyright $($ ) 1982, with permission from Elsevier. ${ }^{19}$ 
Table 2 Patient characteristics

\begin{tabular}{ll}
\hline Characteristics & Patients, n (\%) \\
\cline { 2 - 2 } & OR, median (range) \\
\hline Age, median (range) years & $72(32.5-97.3)$ \\
Breslow thickness (mm) & $1.7(0.4-10.0)$ \\
Gender & \\
Male & $6(30)$ \\
Female & $14(70)$ \\
Stage (AJCC) & \\
IIIb & II (55) \\
IIIc & $8(40)$ \\
IV & I (5) \\
Limb & \\
Upper & $1(5)$ \\
Lower & $19(95)$ \\
Site of disease & \\
Below knee & $4(20)$ \\
Above knee & $4(20)$ \\
Below/above knee & II (55) \\
Below elbow & I (5) \\
Lesions (n) & \\
$0-5$ & $3(15)$ \\
$5-10$ & $3(15)$ \\
I0-I5 & $2(10)$ \\
I5-20 & $2(10)$ \\
$>20$ & $4(20)$ \\
Not determined & $6(30)$ \\
\hline Ab &
\end{tabular}

Abbreviations: AJCC, American Joint Committee on Cancer; OR, odds ratio.

patients received two ILI procedures, and three had triple ILI procedures. The relevant patient and tumor data are shown in Table 2. Of the 20 patients who received ILI therapy, 19 involved the lower limb and one ILI was performed on the upper limb.

The median follow-up duration after the initial ILI for all 20 patients was 15.9 (2.7-121.4) months. The patient population consisted of 14 women and six men of median age 72.6 (32.4-97.2) years. Computed tomography scans were performed at the time of ILI. All patients had either local recurrences or in-transit melanoma metastases in the limb, of whom 11 had no lymph node involvement, eight had previous regional lymph node involvement, and one had evidence of possible small pulmonary metastases.

The median volume infused into lower limb tissue was 6.75 (4.0-15.5) L, while the one case involving the upper limb had an infusion volume of $2.0 \mathrm{~L}$. The median melphalan dose administered was $7.5(6.0-13.3) \mathrm{mg}$ per liter of tissue and the median actinomycin D dose was $50.0(36.3-67.5) \mu \mathrm{g}$ per liter of tissue. Median initial intramuscular and subcutaneous limb temperatures were $35.1^{\circ} \mathrm{C}\left(32.5^{\circ} \mathrm{C}-38.0^{\circ} \mathrm{C}\right)$ and $34.5^{\circ} \mathrm{C}\left(19.4^{\circ} \mathrm{C}-36.4^{\circ} \mathrm{C}\right)$, respectively, with median final temperatures of $34.9^{\circ} \mathrm{C}\left(28.7^{\circ} \mathrm{C}-39.0^{\circ} \mathrm{C}\right)$ and $34.9^{\circ} \mathrm{C}$ $\left(21.8^{\circ} \mathrm{C}-38.2^{\circ} \mathrm{C}\right)$, respectively.

\section{Initial single ILI outcomes}

\section{Toxicity}

There was one case of grade I limb toxicity (5\%), 14 cases of grade II limb toxicity (70\%), three cases of grade III limb toxicity (15\%), and two cases of grade IV limb toxicity (10\%). No cases of grade $\mathrm{V}$ limb toxicity requiring limb amputation were observed. Of the two grade IV cases, one was considered to be a chemical cellulitis, which resolved without intervention. The other patient experienced severe local chemotherapy toxicity around the site of the catheter tips due to poor venous drainage subsequent to prior deep venous thrombosis years previously, but this was not declared until after the procedure, and required extensive debridement and grafting (longest bed stay). The median length of hospital stay for all patients was 8.5 (6-18) days.

\section{Clinical responses}

After clinical evaluation, there was an overall response rate of $70 \%$, with a complete response rate of $20 \%$ and a partial response rate of $50 \%$. Four patients were classified as having stable disease (20\%), and if these were included, the overall "clinically significant" response rate was $90 \%$. Another two patients were classed as having progressive disease (10\%). In the four patients who achieved a complete response, only one has since developed further systemic disease; the median time from ILI until progression was 18.2 (4.1-45.7) months. Three of the four patients who achieved a complete response had regional lymph node involvement and nodal surgery prior to ILI and did not experience any recurrence. The other patient who had a complete response in the infused limb had stage IV disease, but did not experience limb recurrence. Of the ten patients who achieved a partial response, all but two have developed progressive or new disease, occurring after a median follow-up period of $7.3(2.0-12.0)$ months after ILI. Of those ten patients with a partial response, eight were classified as having local or in-transit disease without regional nodal involvement, whilst the other two had regional nodal disease.

\section{Overall final ILI outcomes}

\section{Toxicity}

Limb toxicities after the final ILI for all patients $(\mathrm{n}=20)$ were: grade $1,5 \%(\mathrm{n}=1)$; grade $2,75 \%(\mathrm{n}=15)$; grade 3 , $10 \%(\mathrm{n}=2)$; and grade $4,10 \%(\mathrm{n}=2)$.

\section{Clinical responses}

After the final ILI for all patients, there was an overall response rate of $70 \%$, with a complete response rate of $35 \%$ $(n=7)$ and a partial response rate of $35 \%(n=7)$. Stable disease was seen in $20 \%$ of cases $(n=4)$ and progressive 
disease in $10 \%(n=2)$. If stable disease was included, the overall "clinically significant" response rate was $90 \%$.

\section{Outcomes in patients receiving only one ILI procedure Toxicity}

Limb toxicities for this patient subset $(n=15)$ were: grade 1 , $6.7 \%(\mathrm{n}=1)$; grade $2,73.3 \%(\mathrm{n}=11)$; grade $3,6.7 \%(\mathrm{n}=1)$; and grade $4,13.3 \%(n=2)$.

\section{Clinical responses}

The overall response rate for patients who received one ILI alone was $66.6 \%(\mathrm{n}=15)$, with a complete response rate of $26.6 \%(n=4)$, a partial response rate of $40 \%(n=6)$, stable disease in $26.6 \%(n=4)$, and progressive disease in one patient $(6.8 \%)$. If stable disease was included, the overall “clinically significant" response rate was $93.2 \%$.

\section{Assessment of all ILI therapy episodes for overall toxicity}

Limb toxicity following all ILI procedures $(n=28)$ was: grade $1,3.6 \%(\mathrm{n}=1)$; grade $2,71.4 \%(\mathrm{n}=20)$; grade 3, $17.8 \%(\mathrm{n}=5)$; and grade $4,7.2 \%(\mathrm{n}=2)$. Of the 28 procedures, 19 (67.9\%) showed raised creatine kinase levels postoperatively. The median duration until peak creatine kinase was four (1-6) days, while the median peak level was 680.5 (30-27,650) Units/liter. Ordinal regression showed that the peak creatine kinase level had a statistically significant association with Wieberdink toxicity grade $(P=0.012)$. The peak creatine kinase level did not show any association with clinical response or disease-free interval $(P>0.05)$.

\section{Discussion}

Recurrence of melanoma in a limb represents a spectrum of disease, but is predominantly either cutaneous or subcutaneous, or a combination of both. Involvement of limb bones or muscle is rare, but can occur, sometimes associated with deep and large subcutaneous tumor deposits, and/or enlargement of lymph node metastases in the deep calf, popliteal, or epitrochlear regions. Bulky disease may occur if superficial deposits enlarge and/or become confluent. Essentially all cutaneous and subcutaneous recurrent melanoma arises from lymphatic metastatic spread that is in-transit to the regional lymph nodal basin. This fact was recognized in the 2001 and 2009 American Joint Committee on Cancer staging classification, where intransit lesions were classified as stage III melanoma. ${ }^{21}$ Despite this, local surgical resection of superficial limb deposits, in small numbers or appearing sequentially, is often surprisingly effective for control of recurrent disease of the limb, even in the longer term. However, there are difficult situations where melanoma metastases develop inexorably in a limb and are not amenable to standard local therapies. ${ }^{22}$ Systemic chemotherapy is usually ineffective for treatment of advanced melanoma recurrence in a limb, and radiotherapy may have an effect, but this is often unpredictable. ${ }^{23}$ Recent therapies, such as B-raf inhibitory antibodies, can have some effect, although this is often temporary or short-lived. Over half of all melanoma patients are B-raf mutant-negative and do not show responsiveness. About half of B-raf-positive patients show responses, but most of these eventually fail. Also, dose-limiting adverse events occur in about $40 \%$ of patients treated with B-raf. ${ }^{24,25}$

Regional chemotherapy for locally recurrent or in-transit metastatic melanoma distal to the axilla or groin has been shown to be an effective treatment option in many situations where surgical resection is not possible or has failed. ${ }^{13,18}$ Isolated limb perfusion has been used extensively internationally, whereas ILI has become widely used by specialist melanoma centers in Australia, with gradual interest internationally. ${ }^{26,27}$ The advantages of ILI are its technical simplicity and practicality, good tolerability in patients (especially the elderly), and the relative ease of repeating the procedure(s). ILI also has a relatively low complication rate compared with isolated limb perfusion, and does not require specialized extracorporeal bypass facilities, invasive surgery, or blood transfusion. ${ }^{18}$ Further, the reduced operative time and cost required per procedure makes it economically more attractive for health budgets than isolated limb perfusion. Lower rates of limb toxicity and morbidity, including compartment syndrome, peripheral neuropathy, infection, and venous thrombosis, are also significant additional economic advantages. ${ }^{17}$

The current analysis shows that the patients treated at the Adelaide Melanoma Unit experience very low levels of limb toxicity, with only one significant long-term complication recorded so far. However, this occurred in a patient with a specific venous stasis problem that was only reported after ILI. Our overall toxicity rates assessing each ILI episode as an independent event $(\mathrm{n}=28)$ demonstrated relatively low morbidity ( $75 \%$ Wieberdink grade 1 or 2$)$. For overall outcomes $(\mathrm{n}=20)$ after the final ILI, the toxicity profile (grade 1 , $5 \%$; grade $2,75 \%$; grade $3,10 \%$; grade $4,10 \%$ ) was not significantly different from either independently analyzed ILI procedures or first ILI procedures. This indicates that the post-ILI toxicity profile remained similar regardless of the number of procedures that were performed per patient, which is in accordance with previous reports on repeat ILI. ${ }^{28}$ The median follow-up period after initial ILI for all 20 patients was 15.9 (2.7-121.4) months, providing a good indication that successful longer-term results can be achieved. 
After the final ILI, there was an overall clinical response rate of $70 \%$, with a complete response rate of $35 \%$ and a partial response rate of $35 \%$. Stable disease was seen in $20 \%$ and progressive disease in $10 \%$ of patients. If stable disease was included, the overall "clinically effective" response rate was $90 \%$, where any clinically significant or meaningful measurable response was observed.

Our response rates compare favorably with previously published ILI studies. At the time of publication, the largest ILI study, performed by the Sydney Melanoma Unit, consisted of 185 patients. ${ }^{17}$ That study showed an overall response rate of $84 \%$ (complete response $38 \%$, partial response $46 \%$ ) using a technique similar to that used in this analysis after one ILI. After repeated ILI procedures $(n=48)$, the overall response was $83 \%$ (complete response $23 \%$ and partial response $60 \%) .{ }^{28}$ The first and to date only multicenter study investigating the ILI procedure showed a slightly lower but still satisfactory response rate in comparison with the Sydney Melanoma Unit study with an overall response rate of $64 \%$ (complete response $31 \%$, partial response $33 \%$ ), after one or more ILI procedures. ${ }^{26}$

Measurement of visible melanoma nodules or deposits was performed with good reproducibility by measuring in two directions perpendicular to each other. However, for deeper deposits, imaging was required and this was done by either Doppler ultrasound or computed tomography scanning. An alternative would be to use contrast-enhanced ultrasound or dynamic contrast-enhanced ultrasonography for evaluations using microbubble contrast. ${ }^{29}$ However, the most ideal lesions for dynamic contrast-enhanced ultrasonography are those that are over $2 \mathrm{~cm}$ in size, with sufficient necrosis and more vascularization. Smaller deposits can be more difficult to evaluate, and were the main type of in-transit deposit encountered in our study.

ILI chemotherapy could be combined with approved or experimental systemic therapies, such as interleukin-2, standard chemotherapy, radiotherapy, or serine-threonine protein kinase B-Raf inhibitory antibodies (B-Raf), Cytotoxic T lymphocyte associated antigen-4 (CTLA-4), mitogen-activated protein kinase kinase (MEK) Programmed cell Death Receptor-1 (PD-1), Programmed cell Death Ligand-1 (PDL1) inhibitor therapy, or local intra-lesional therapies such as coxsackie virus or rose bengal, and be practice-changing. ILI using non-cytotoxic agents, perhaps including some of those above, might be explored.

Although ILI is essentially a regional local treatment confined to the melanoma-affected limb, long-term survival is not uncommon. This raises the distinct possibility of systemic immune modulation from "autovaccination" events as a result of tumor necrosis induced by ILI chemotherapy, which has been proposed previously. ${ }^{30}$ Similar reports have been made for other local therapies associated with direct lesional injection of melanoma metastases, and topical desensitising therapies such as diphencyprone (DPCP), where "bystander"-type regressions have been noted in noninjected metastases. ${ }^{31-34}$

\section{Conclusion}

The current analysis of our procedures indicates that ILI is a particularly useful method for controlling metastatic limb melanoma, with high efficacy and results comparable with those reported using isolated limb perfusion chemotherapy regimens, avoiding a more invasive and costly procedure. ILI offers the advantage of being a relatively simple method with lower morbidity. Our findings validate previous studies using ILI. Larger multicenter studies are required to investigate the true efficacy of ILI for treating locally advanced melanoma confined to the limb. Further work on the ILI method is currently required to improve and refine this promising technique for treatment of limb melanoma. Long-term survivors are reported after ILI. Combination therapy using ILI chemotherapy and other systemic therapies may be a useful future direction.

\section{Acknowledgments}

The authors wish to thank Professor John F Thompson, Sydney Melanoma Unit, Royal Prince Alfred Hospital, Sydney, for his guidance and advice in the early stages of establishment of ILI therapy, and Dr R Waugh, Radiology Department, Royal Prince Alfred Hospital, for his advice regarding catheter selection and technique. Thanks are also extended to Dr G Freer for his anesthetic expertise in pioneering the ILI procedure at our institution. Additional thanks for statistical support go to Nancy Briggs and Tom Sullivan of the Department of Public Health, The University of Adelaide, and to our radiological colleagues at the Royal Adelaide Hospital for their catheter expertise. We thank Hidde Kroon (The Netherlands) for reviewing the manuscript.

\section{Disclosure}

The authors report no conflicts of interest in this work.

\section{References}

1. Australian Institute of Health and Welfare and Australasian Association of Cancer Registries 2012. Cancer in Australia: an overview, 2012. Australian Cancer Incidence and Mortality Workbooks Melanoma. Cancer series no 74. Catalog number CAN 70. Canberra, Australia: Australian Institute of Health and Welfare; 2012. 
2. Francken AB, Accortt NA, Shaw HM, et al. Prognosis and determinants of outcome following locoregional or distant recurrence in patients with cutaneous melanoma. Ann Surg Oncol. 2008;15:1476-1484.

3. Creech O Jr, Krementz ET, Ryan RF. Chemotherapy of melanoma of the extremities by isolated perfusion. Cancer Chemother Rep. 1962;16: 579-581.

4. Hayes AJ, Neuhaus SJ, Clark MA, Thomas JM. Isolated limb perfusion with melphalan and tumor necrosis factor alpha for advanced melanoma and soft-tissue sarcoma. Ann Surg Oncol. 2007;14:230-238.

5. Knorr C, Meyer T, Janssen T, et al. Hyperthermic isolated limb perfusion (HILP) in malignant melanoma. Experience with 101 patients. Eur $J$ Surg Oncol. 2006;32:224-227.

6. Noorda EM, Vrouenraets BC, Nieweg OE, Kroon BB. Isolated limb perfusion in regional melanoma. Surg Oncol Clin N Am. 2006;15: 373-384.

7. Takkenberg RB, Vrouenraets BC, van Geel AN, et al. Palliative isolated limb perfusion for advanced limb disease in stage IV melanoma patients. J Surg Oncol. 2005;91:107-111.

8. Aloia TA, Grubbs E, Onaitis M, et al. Predictors of outcome after hyperthermic isolated limb perfusion: role of tumor response. Arch Surg. 2005;140:1115-1120.

9. Thompson JF, Hunt JA, Shannon KF, Kam PC. Frequency and duration of remission after isolated limb perfusion for melanoma. Arch Surg. 1997;132:903-907.

10. Reintgen M, Reintgen C, Nobo C, Giuliano Shivers RS, Reintgen D. Regional therapy for recurrent metastatic melanoma confined to the extremity: hyperthermic isolated limb perfusion vs isolated limb infusion. Cancers. 2012;2:43-50.

11. Deroose JP, Eggermont AM, van Geel AN, Verhoef C. Isolated limb perfusion for melanoma in-transit metastases: developments in recent years and the role of tumor necrosis factor alpha. Curr Opin Oncol. 2011;23:183-188.

12. Testori A, Verhoef C, Kroon HM, et al. Treatment of melanoma metastases in a limb by isolated limb perfusion and isolated limb infusion. J Surg Oncol. 2011;104:397-404.

13. Vrouenraets BC, Kroon BB, Nieweg OE, Thompson JF. Isolated limb perfusion for melanoma: results and complication. In: Thompson JF, Morton DL, Kroon BB, editors. Textbook of Melanoma. London, UK: Martin Dunitz; 2004.

14. Koops HS, Vaglini M, Suciu S, et al. Prophylactic isolated limb perfusion for localized, high-risk limb melanoma: results of a multicenter randomized phase III trial. European Organization for Research and Treatment of Cancer Malignant Melanoma Cooperative Group Protocol 18832, the World Health Organiation Melanoma Program Trial 15, and the North American Perfusion Group Southwest Oncology Group-8593. J Clin Oncol. 1998;16:2906-2912.

15. Thompson JF, Kam PC, Waugh RC, Harman CR. Isolated limb infusion with cytotoxic agents: a simple alternative to isolated limb perfusion. Semin Surg Oncol. 1998;14:238-247.

16. Lindner P, Doubrovsky A, Kam PC, Thompson JF. Prognostic factors after isolated limb infusion with cytotoxic agents for melanoma. Ann Surg Oncol. 2002;9:127-136.
17. Kroon HM, Moncrieff M, Kam PC, Thompson JF. Outcomes following isolated limb infusion for melanoma. A 14-year experience. Ann Surg Oncol. 2008;15:3003-3013.

18. Kroon HM, Thompson JF. Isolated limb infusion: a review. J Surg Oncol. 2009;100:169-177.

19. Wieberdink J, Benckhuysen C, Braat RP, et al. Dosimetry in isolation perfusion of the limbs by assessment of perfused tissue volume and grading of toxic tissue reactions. Eur J Cancer Clin Oncol. 1982;18: 905-910.

20. World Health Organization. WHO Handbook for Reporting Results of Cancer Treatment. Geneva, Switzerland: World Health Organization; 1979.

21. Balch CM, Buzaid AC, Soong SJ, et al. Final version of the American Joint Committee on Cancer staging system for cutaneous melanoma. J Clin Oncol. 2001;19:3635-3648.

22. Testori A, Faries MB, Thompson JF, et al. Local and intralesional therapy of in-transit melanoma metastases. J Surg Oncol. 2011;104:391-396.

23. Hong A, Fogarty G. Role of radiation therapy in cutaneous melanoma. Cancer J. 2012;18:203-207.

24. Chapman PB, Hauschild A, Robert C, et al. Improved survival with vemurafenib in melanoma with BRAF V600E mutation. $N$ Engl J Med. 2011;364:2507-2516

25. Sosman JA, Kim KB, Schuchter L, et al. Survival in BRAF V600mutant advanced melanoma treated with vemurafenib. $N$ Engl J Med. 2012;366:707-714.

26. Beasley GM, Caudle A, Petersen RP, et al. A multi-institutional experience of isolated limb infusion: defining response and toxicity in the US. J Am Coll Surg. 2009;208:706-715.

27. Duprat Neto JP, Mauro AC, Molina AS, et al. Isolated limb infusion with hyperthermia and chemotherapy for advanced limb malignancy: factors influencing toxicity. ANZ J Surg. September 24, 2012. [Epub ahead of print.]

28. Kroon HM, Lin DY, Kam PC, Thompson JF. Efficacy of repeat isolated limb infusion with melphalan and actinomycin D for recurrent melanoma. Cancer. 2009;115:1932-1940.

29. Lassau N, Chami L, Chebil M, et al. Dynamic contrast-enhanced ultrasonography (DCE-US) and anti-angiogenic treatments. Discov Med. 2011;11:18-24.

30. Coventry BJ, Ashdown ML. Complete clinical responses to cancer therapy caused by multiple divergent approaches: a repeating theme lost in translation. Cancer Manag Res. 2012;4:137-149.

31. Thompson JF, Hersey P, Wachter E. Chemoablation of metastatic melanoma using intralesional rose bengal. Melanoma Res. 2008;18: 405-411.

32. Foote MC, Burmeister BH, Thomas J, Mark Smithers B. A novel treatment for metastatic melanoma with intralesional rose bengal and radiotherapy: a case series. Melanoma Res. 2010;20:48-51.

33. Shafren DR, Au GG, Nguyen T, et al. Systemic therapy of malignant human melanoma tumors by a common cold-producing virus, coxsackievirus A21. Clin Cancer Res. 2004;10:53-60.

34. Damian DL, Shannon KF, Saw RP, Thompson JF. Topical diphencyprone immunotherapy for cutaneous metastatic melanoma. Australasian Journal of Dermatology. 2009;50:266-271.
Cancer Management and Research

\section{Publish your work in this journal}

Cancer Management and Research is an international, peer-reviewed open access journal focusing on cancer research and the optimal use of preventative and integrated treatment interventions to achieve improved outcomes, enhanced survival and quality of life for the cancer patient The journal welcomes original research, clinical \& epidemiological

\section{Dovepress}

studies, reviews \& evaluations, guidelines, expert opinion \& commentary, case reports \& extended reports. The manuscript management system is completely online and includes a very quick and fair peerreview system, which is all easy to use. Visit http://www.dovepress.com/ testimonials.php to read real quotes from published authors. 